\title{
DESEMPENHO SAZONAL DA EVAPOTRANSPIRAÇÃO DE REFERÊNCIA EM AQUIDAUANA, MS
}

\author{
Everton Rossi Rigoni ${ }^{1}$, Gabriel Queiroz de Oliveira², Guilherme Augusto Biscaro ${ }^{3}$, Marcos Vinnícius Braga \\ Machado de Queiróz ${ }^{4}$, Adriano da Silva Lopes ${ }^{5}$
}

\begin{abstract}
RESUMO
O presente estudo teve como objetivo, determinar a evapotranspiração de referência, avaliando-se a precisão dos métodos empíricos Benevides-Lopez, Camargo, FAO-24 Radiação, Hamon, Hargreaves-Samani, Klarrufa, JesenHaise, Makkink, Prietley-Taylor e Turc, comparando com o método padrão Penman-Monteith FAO-56, em diferentes épocas do ano em Aquidauana, MS. Este trabalho foi realizado com auxílio dos dados meteorológicos coletados diariamente entre o período de 01 de janeiro de 2008 a 31 dezembro de 2011 na rede de estações do Instituto Nacional de Meteorologia (INMET), situado no município de Aquidauana-MS. Foram avaliados valores diários da estimativa da evapotranspiração de referência (ETo), em mm dia $\mathrm{m}^{-1}$, para a época de verão, outono, inverno e primavera, utilizandose métodos empíricos e o de Penman-Monteith, parametrizado pela FAO, no qual serviu de modelo de comparação. Foram determinados e considerados na avaliação dos métodos a média da ETo, o coeficiente de determinação $\left(\mathrm{r}^{2}\right)$, correlação (r) e de erro padrão de estimativa (EPE). Para a avaliação da exatidão dos métodos avaliados, foram determinados o índice de concordância (d) e o índice de desempenho (c). Diante da série de dados, conclui-se que os métodos Priestley-Taylor e Radiação Solar atendem satisfatoriamente à estimativa da evapotranspiração de referência em Aquidauana-MS, para todas as épocas do ano.
\end{abstract}

Palavras-chave: agrometeorologia, Penman-Monteih, região Cerrado-Pantanal, Mato Grosso do Sul

\section{ABSTRACT \\ SEASONAL PERFORMANCE OF THE REFERENCE EVAPOTRANSPIRATION IN AQUIDAUANA, MATO GROSSO DO SUL STATE, BRAZIL}

The aim of this study was to determine the reference evapotranspiration by assessing the accuracy of empirical methods Benevides-Lopez, Camargo, FAO-24 Radiation, Hamon, Hargreaves-Samani, Klarrufa, Jesen-Haise, Makkink, Prietley-Taylor, and Turc, comparing with the Penman-Monteith FAO-56 in different seasons in Aquidauana, Mato Grosso do Sul State, Brazil. This work was done with the collected daily weather data obtained between the period from 01 January 2008 to 31 December 2011 in the network of stations of the Instituto Nacional de Meteorologia (National Institute of Meteorology), located in Aquidauna-MS. Were evaluated daily values of reference evapotranspiration (ETo) in mm day ${ }^{-1}$ for the summer period, autumn, winter and spring, using empirical methods and Penman-Monteith, parameterized by FAO in which he served as a model for comparison. Were determined and considered in theevaluation of methods the average ETo, the coefficient of determination $\left(r^{2}\right)$, correlation ( $r$ ) and standard error of estimate (SEE). To evaluate the accuracy of the methods evaluated were determined the level of agreement (d), and the performance index (c). Given the number of data, it is concluded that the methods Priestley-Taylor and Solar Radiation cater satisfactorily to estimate reference evapotranspiration in Aquidauna-MS for all seasons.

Keywords: agrometeorology, Penman-Monteih, Cerrado Pantanal region, Mato Grosso do Sul State

Recebido para publicação em 28/03/2013. Aprovado em 04/11/2013.

1 - Eng. Agrônomo, Mestre em Agronomia, FCA/UFGD, Dourados, MS. e_rigoni@hotmail.com

2 - Eng. Agrônomo, Doutorando em Agronomia, FCA/UFGD, Dourados, MS. gabrielqo@hotmail.com

3 - Eng. Agrícola, Professor Adjunto, FCA/UFGD, Dourados, MS. guilhermebiscaro@ufgd.edu.br

4 - Eng. Agrônomo, Mestrando em Engenharia Agrícola, FCA/UFGD, Dourados, MS. marcosbrag@hotmail.com

5 - Eng. Agrônomo Professor Adjunto, UUA/UEMS, Aquidauana, MS. lopes@uems.br 


\section{INTRODUÇ̃̃O}

A evapotranspiração é uma fração do ramo superficial ascendente do ciclo hidrológico, forma pela qual a água da superfície terrestre passa para a atmosfera no estado de vapor, sendo a resposta aos processos evaporativos do solo (nu ou vegetado) juntamente com o respiratório dos vegetais, tendo papel importantíssimo no ciclo hidrológico em termos globais e regionais (SILVA; SOUZA, 2011). Dentre os vários tipos de evapotranspiração, destaca-se a evapotranspiração de referência (ETo), por permitir e facilitar a estimativa da evapotranspiração da cultura (ETc). A ETo é uma variável relevante para o planejamento de irrigação, pode ser afetada principalmente pelos fatores climáticos como, temperatura, umidade relativa do ar, velocidade do vento, etc. (SOUSA et al., 2010).

$\mathrm{Na}$ agricultura, informações quantitativas da evapotranspiração são de grande importância para a avaliação da severidade, distribuição e frequência dos períodos de seca, assim como, elaboração de projetos e manejo de sistemas de irrigação e drenagem (HENRIQUE; DANTAS, 2007). Assim, as informações sobre a ETo, que levam a estimativas da ETc, tornam-se ferramentas importantes no manejo da irrigação (ARAÚJO et al., 2007).

O Boletim da FAO 56 (United Nations, Food and Agricultural Organization) define a ETo como sendo a evapotranspiração de uma cultura hipotética, com altura fixa de $0,12 \mathrm{~m}$, albedo igual a 0,23 e resistência da cultura ao transporte de vapor d'água igual a $70 \mathrm{~s} \mathrm{~m}^{-1}$. A cultura hipotética está relacionada a uma superfície gramada, de altura uniforme, em crescimento ativo, cobrindo totalmente a superfície do solo e sem restrição hídrica (ALLEN et al., 1998).

Existem diversos métodos na literatura para se estimar a evapotranspiração de referência utilizando-se parâmetros climatológicos. Dentre eles, podem-se citar Penman-Monteith, BenevidesLopez, Camargo, FAO-24 Radiação, Hamon, Hargreaves-Samani, Klarrufa, Jesen-Haise, Makkink, Prietley-Taylor, Turc e muitos outros. Porém, esses métodos foram desenvolvidos nas mais diversas condições climáticas e de manejo de culturas, sendo necessária a calibração dos mesmos para aplicação em regiões de climas diferentes do qual foram desenvolvidos.

Diversos pesquisadores em todo o mundo propuseram modelos indiretos para a estimativa da ETo, com as mais diferentes concepções e número de variáveis envolvidas. Antes de se eleger o modelo a ser utilizado para a estimativa da ETo, é necessário saber quais os elementos climáticos disponíveis; a partir daí, verifica-se quais podem ser aplicados, uma vez que a utilização dos diferentes métodos para certo local de interesse fica na dependência dessas variáveis (ARAÚJO et al., 2007). As conclusões variam muito segundo cada autor, principalmente, pelas áreas de estudos serem climatologicamente diferentes. Desta forma, Pereira et al. (2009) recomendam que antes de aplicar um método para determinado local, é necessário verificar o desempenho deste e, quando necessário, fazer calibrações a fim de minimizar erros de estimativa, sendo a equação PenmanMonteith FAO-56 a referência padrão para esses ajustes. Essa aplicação deve ser feita para locais próximos que tenham medidas climáticas suficientes (SMITH, 1991).

Desta forma, o objetivo deste trabalho foi determinar o desempenho sazonal da ETo, avaliando-se a precisão dos métodos empíricos que se baseia na ausência da radiação solar: BenevidesLopez, Camargo, FAO-24 Radiação, Hamon, Hargreaves-Samani, Klarrufa e os métodos que utilizam a radiação solar: Jesen-Haise, Makkink, Prietley-Taylor, e Turc, comparando com o método padrão Penman-Monteith FAO-56, para os anos de 2008 a 2011 na região de Aquidauana, MS.

\section{MATERIAL E MÉTODOS}

O presente trabalho foi realizado com auxílio dos dados diários de: temperatura do ar máxima (Tmax) e mínima do ar (Tmin), temperatura do ponto de orvalho máxima $\left(\mathrm{T}_{\mathrm{po}} \max \right)$ e mínima ( $\left.\mathrm{T}_{\mathrm{po}} \mathrm{min}\right)$, umidade relativa máxima do ar (URmax) e mínima do ar (URmin), velocidade do vento a $2 \mathrm{~m}$ de altura $\left(\mathrm{U}_{2}\right)$ e radiação solar global (Rs) (Quadro 1), obtidos entre o período de 01 de janeiro de 2008 a 31 dezembro de 2011, da estação meteorológica automática do município de Aquidauana-MS $\left(20^{\circ} 20^{\prime}\right.$ Sul, $55^{\circ} 48^{\prime}$ 'Oeste e altitude de $155 \mathrm{~m}$ acima do nível mar), obtidos na rede de estações do Instituto Nacional de Meteorologia (INMET).

O território do município de AquidauanaMS possui características do bioma de Cerrado e Pantanal (Figura 1). O clima da região é classificado, segundo Köppen, como do tipo Aw, definido como clima tropical quente sub-úmido, com estação

\section{REVENG}


chuvosa no verão e seca no inverno e precipitação pluviométrica anual média de $1.200 \mathrm{~mm}$. O período chuvoso se estende de outubro a março; entre abril e setembro, tem-se o período seco, com os menores índices de precipitação, ocorrendo entre julho a agosto (ZAVATTINI, 2009).

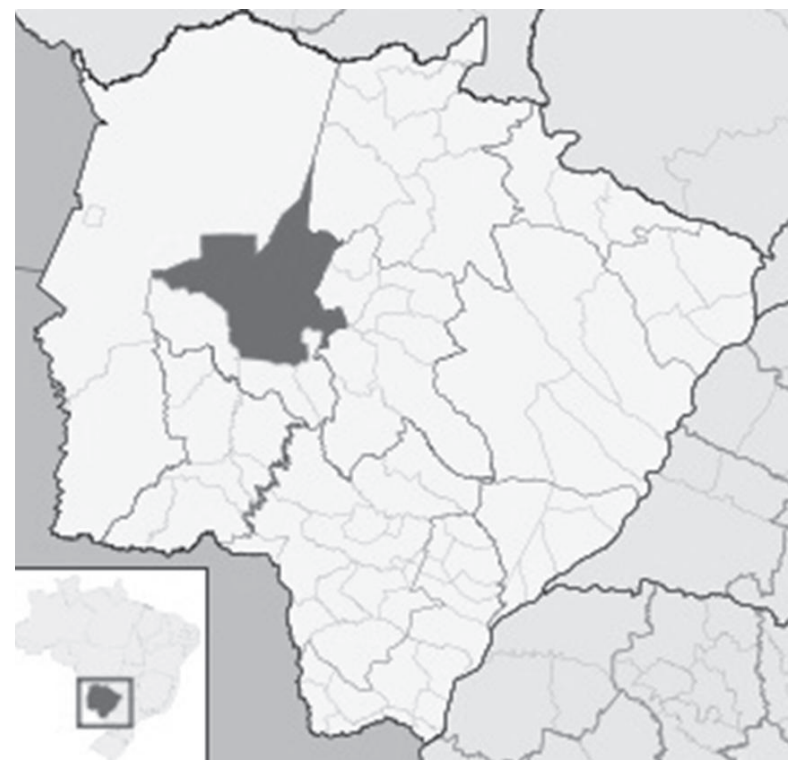

Figura 1. Localização geográfica do Estado de Mato Grosso do Sul (MS) no mapa do Brasil (abaixo) e da cidade de Aquidauana no mapa do MS.
Foram avaliados valores diários da estimativa da ETo, em mm dia ${ }^{-1}$, para os períodos Solstício de Verão (21 de dezembro a 20 de Março), Equinócio de Outono (21 de março a 20 de Junho), Solstício de Inverno (21 de junho a 22 de setembro) e Equinócio de Primavera (23 de setembro a 20 de dezembro), utilizando-se métodos empíricos e o de Penman-Monteith, parametrizado pela FAO no qual serviu de modelo de comparação (ALLEN et al., 1998) (Equação 1).

$\mathrm{ETo}_{(\mathrm{PM})}=\frac{0,408 \Delta(\mathrm{Rn}-\mathrm{G})+\gamma\left(\frac{900 \mathrm{U}_{2}}{\mathrm{~T}_{\text {med }}+273}\right)(\text { es - ea })}{\Delta+\gamma\left(1+0,34 \mathrm{U}_{2}\right)}$

em que,

$\mathrm{ETo}_{(\mathrm{PM})}=$ evapotranspiração de referência pelo método PM, (mm dia $\left.{ }^{-1}\right)$;

$\mathrm{Rn}=$ saldo de radiação, $\left(\mathrm{MJ} \mathrm{m}^{-2} \mathrm{dia}^{-1}\right)$;

$\mathrm{G}=$ fluxo de calor no solo, $\left(\mathrm{MJ} \mathrm{m}^{-2} \mathrm{dia}^{-1}\right)$;

$\Delta=$ declinação da curva de saturação do vapor da água, $\left(\mathrm{kPa}^{\circ} \mathrm{C}^{-1}\right)$;

$\mathrm{U}_{2}=$ velocidade média do vento a $2 \mathrm{~m}$ acima da superfície do solo, $\left(\mathrm{m} \mathrm{s}^{-1}\right)$;

$\mathrm{T}=$ temperatura média do ar, $\left({ }^{\circ} \mathrm{C}\right)$;

$\mathrm{e}_{\mathrm{s}}=$ pressão de saturação de vapor, $(\mathrm{kPa})$;

$\mathrm{e}_{\mathrm{a}}=$ pressão atual de vapor, $(\mathrm{kPa}) ; \gamma=$ constante psicrométrica, $\left(\mathrm{kPa}^{\circ} \mathrm{C}^{-1}\right)$.

Quadro 1. Valores de Tmax e Tmin, $T_{p 0} \max$ e $T_{p o} \min , U R \max$ e URmin, $U_{2}$ e Rs de janeiro de 2008 a dezembro de 2011, em Aquidauana, MS

\begin{tabular}{|c|c|c|c|c|c|c|c|c|}
\hline \multirow{2}{*}{ Mês } & Tmax & Tmin & $\mathrm{T}_{\mathrm{p} 0} \max$ & $\mathrm{T}_{\mathrm{p} 0} \min$ & URmax & URmin & \multirow{2}{*}{$\frac{\mathrm{U}_{2}}{\mathrm{~m} \mathrm{~s}^{-1}}$} & \multirow{2}{*}{$\begin{array}{c}\mathrm{Rs} \\
\mathrm{MJ} \mathrm{m}^{-2} \mathrm{dia}^{-1}\end{array}$} \\
\hline & \multicolumn{4}{|c|}{${ }^{\circ} \mathrm{C}$} & \multicolumn{2}{|c|}{$\%$} & & \\
\hline JAN & 32,33 & 22,69 & 24,75 & 20,01 & 93,35 & 55,84 & 1,47 & 21,94 \\
\hline FEV & 33,32 & 23,09 & 24,79 & 20,05 & 92,78 & 52,92 & 1,41 & 23,65 \\
\hline MAR & 33,61 & 22,25 & 24,42 & 19,09 & 92,92 & 48,95 & 1,22 & 23,33 \\
\hline $\mathrm{ABR}$ & 32,74 & 19,37 & 22,06 & 15,57 & 91,31 & 43,13 & 1,25 & 20,09 \\
\hline MAI & 28,91 & 16,31 & 19,75 & 13,50 & 93,11 & 48,68 & 1,26 & 16,67 \\
\hline JUN & 28,21 & 15,11 & 18,98 & 12,39 & 91,97 & 46,53 & 1,24 & 14,18 \\
\hline JUL & 29,91 & 15,15 & 18,26 & 10,58 & 88,27 & 38,61 & 1,36 & 17,18 \\
\hline AGO & 31,76 & 16,01 & 17,98 & 10,27 & 83,89 & 33,97 & 1,50 & 17,28 \\
\hline SET & 32,10 & 18,57 & 18,83 & 11,91 & 79,81 & 37,26 & 1,93 & 19,37 \\
\hline OUT & 33,06 & 20,72 & 22,22 & 16,37 & 88,48 & 44,48 & 1,65 & 20,38 \\
\hline NOV & 33,56 & 21,59 & 23,85 & 17,66 & 90,98 & 47,52 & 1,58 & 23,23 \\
\hline DEZ & 33,99 & 22,96 & 24,20 & 18,74 & 90,14 & 49,62 & 1,55 & 25,66 \\
\hline Média & 31,96 & 19,48 & 21,67 & 15,51 & 89,75 & 45,63 & 1,45 & 20,25 \\
\hline
\end{tabular}


Os métodos analisados foram o de BenevidesLopez (BENEVIDES; LOPEZ, 1970), Camargo (CAMARGO; SENTELHAS, 1997), FAO24 Radiação (PEREIRA et al., 1997), Hamon, Hargreaves-Samani (PEREIRA et al. 1997), Klarrufa (KLARRUFA, 1985), Jesen-Haise (JENSEN; HAISE 1963), Makkink, PrietleyTaylor, (PEREIRA et al., 1997) e Turc (TURC, 1961).

Os valores estimados da ETo diária para cada um dos 10 métodos foram comparados com os valores de ETo obtidos pelo método PenmanMonteith (FAO-56). Os indicadores estatísticos utilizados para a avaliação da precisão dos modelos foram à análise de regressão linear para determinação dos coeficientes da equação ( $\mathrm{Y}=\mathrm{a}+$ bx) e do coeficiente de determinação e correlação ( $r^{2}$ e $r$ respectivamente) (Equação 2 e 3 ).

$$
\mathrm{r}^{2}=1-\left[\frac{\sum_{\mathrm{i}=1}^{\mathrm{n}}(\mathrm{Pi}-\mathrm{Oi})^{2}}{\sum_{\mathrm{i}=1}^{\mathrm{n}}(\mathrm{Oi}-\mathrm{O})^{2}}\right]
$$

$$
r=\sqrt{r^{2}}
$$

Outro índice estatístico utilizado para comparação dos valores de $\mathrm{ET}_{\mathrm{o}}$ diária obtidos pelos métodos empíricos em comparação ao padrão Penman-Monteith, foi o erro padrão de estimativa (EPE), Equação 4.

$$
E P E=\sqrt{\frac{\sum_{i=1}^{n}(\mathrm{Oi}-\mathrm{Pi})^{2}}{n-1}}
$$

Para avaliar a aproximação entre a $\mathrm{ET}_{\mathrm{o}}$ diária estimada pelos métodos empíricos e a ET ostimada pelo método padrão PM-FAO 56, utilizou-se o índice "d" de concordância ou exatidão proposto por Willmott et al. (1985), onde seus valores variam de "0" (não existe concordância) a "1" (concordância perfeita), Equação 5 e como parâmetro estatístico de comparação, utilizou-se um índice de confiança ou desempenho "c", proposto por Camargo e Sentelhas (1997), reunindo os índices de precisão "r" e de exatidão "d", Equação 6.

$\mathrm{c}=\mathrm{rd}$

$$
\mathrm{d}=1-\left[\frac{\sum_{\mathrm{i}=1}^{\mathrm{n}}(\mathrm{Pi}-\mathrm{Oi})^{2}}{\sum_{\mathrm{i}=1}^{\mathrm{n}}(|\mathrm{Pi}-\overline{\mathrm{O}}|+|\mathrm{Pi}-\overline{\mathrm{O}}|)^{2}}\right]
$$

em que,

$\mathrm{Pi}=$ corresponde aos valores estimados pelos métodos $\left(\mathrm{mm} \mathrm{dia}^{-1}\right)$;

Oi $=$ valores observados pelo método de PenmanMonteith $\left(\mathrm{mm} \mathrm{dia}^{-1}\right)$;

$\mathrm{O}=$ média dos valores observados pelo método de Penman-Monteith $\left(\mathrm{mm} \mathrm{dia}^{-1}\right)$.

$\mathrm{n}=$ número de observações.

O índice de desempenho "c" é interpretado e distribuído nas seguintes classes: péssimo, c $<0,40$; mau, 0,41 < c < 0,50; sofrível, 0,51<c $<0,60$; mediano, $0,61<\mathrm{c}<0,65$; bom, $0,66<\mathrm{c}$ $<0,75$; muito bom, $0,76<\mathrm{c}<0,85$; e ótimo, c $>0,85$ (CAMARGO; SENTELHAS, 1997). Na indicação dos melhores métodos de estimativa da ETo para a região de Aquidauana, MS, foi considerado valores do índice de desempenho "c" superiores a 0,65 . Os cálculos estatísticos foram realizados com o auxílio do software Microsoft Office Excel ${ }^{\circledR}$ versão 2007.

\section{RESULTADOS E DISCUSSÃO}

$\mathrm{Na}$ Figura 2, encontram-se apresentados o coeficiente de determinação $\left(\mathrm{r}^{2}\right)$ entre a ETo estimada pelo método padrão Penman-Monteith e os métodos de Benevides-Lopes, Camargo, FAO-24 Radiação, Hamon, Hargreaves-Samani, Klarrufa, Jensen-Haise, Makink, Priestley-Taylor, e Turc, para o período de verão.

Verifica-se que os métodos não baseados em radiação solar apresentaram baixo coeficiente de determinação $\left(\mathrm{r}^{2}\right)$ com coeficientes angulares (b) próximo de 1, com exceção do método de Klarrufa que por meio da equação de regressão, apresenta tendência em subestimar a ETo em 49,5\%. 

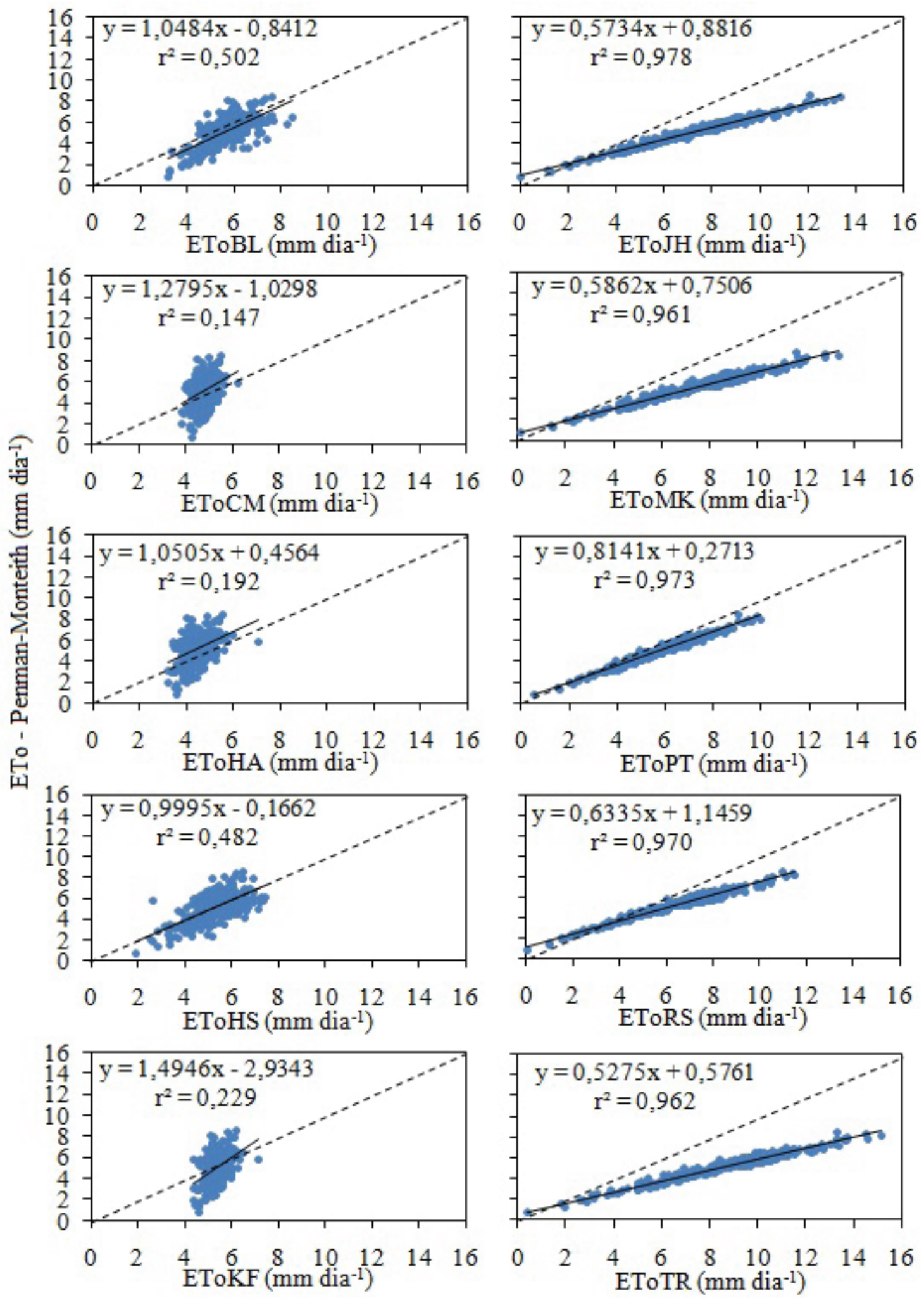

Figura 2. Coeficiente de determinação entre os valores diários de evapotranspiração de referência (ETo) estimados pelo método padrão Penman-Monteith e os métodos empíricos, no solstício de verão de Aquidauana, MS. BL (Benevides-Lopez), CM (Camargo), HA (Hamon), HS (HargreavesSamani), KF (Klarrufa), JH (Jensen-Haise), MK (Makink), PT (Priestley-Taylor), RS (FAO-24 Radiação) e TR (Turc). A linha tracejada indica a reta 1:1. 
Como no período de verão (estação chuvosa) em Aquidauana, MS, apresenta grandes variações de nebulosidade e chuvas, esses fatores climáticos contribuem para mudanças momentânea da temperatura do ar e umidade relativa do ar, que podem em determinado período do dia oscilar bruscamente. Para os métodos baseados na temperatura do ar, os melhores $r^{2}$ neste período foram encontrados pelos métodos de BenevidesLopes e Hargreaves-Samani, que obtiveram um $\mathrm{r}^{2}$ máximo de 0,502 e mínimo de 0,482, respectivamente (Figura 2). Talvez a maior precisão apresentada por esses métodos em relação aos demais métodos, deva-se ao fato de apresentarem na sua equação outros parâmetros climáticos como, por exemplo, umidade relativa do ar, temperaturas máxima, mínima e média do ar, que para efeito, contribuíram para minimizar o efeito das variações da ETo no verão.

Os métodos de estimativa baseados no elemento radiação solar obtiveram estimativa de ETo apresentando um $\mathrm{r}^{2}$ bastante alto (Figura 2), o que indica alta precisão do modelo de regressão. Os seus altos valores de $\mathrm{r}^{2}$ estão diretamente ligados ao uso do parâmetro de radiação solar no método, que é o componente que mais está associado ao fenômeno de evaporação da água. No entanto, verifica-se que o " $b$ " encontra valores afastados de 1. Os métodos FAO-24 Radiação, Jensen-Haise, Makink, Priestley-Taylor e Turc obtiveram "b" na ordem de 0,$573 ; 0,586 ; 0,814 ; 0,634$ e 0,528 , respectivamente (Figura 2). De maneira geral, os métodos de radiação solar, com exceção do método de Priestley-Taylor, estão superestimando a ETo padrão Penman-Monteith em $40 \%$.

O método de Priestley-Taylor apresentou ótimo resultado $(c=0,887)$ por apresentar no seu método o uso da radiação líquida, que é a diferença entre o fluxo de energia de ondas curtas provenientes do sol com a radiação de ondas longas emitidas pela superficie terrestre.

No município de Pesqueira em Pernambuco, Silva e Souza (2011), verificaram que os métodos de Priestley-Taylor e Jensen-Haise, no verão, ajustaram-se com $\mathrm{r}^{2}$ de 0,94 e 0,90 respectivamente, apesar de superestimarem os valores em relação ao método de Penman-Monteith.
No entanto, a adoção do $\mathrm{r}^{2}$ como critério de definição da qualidade de métodos não é adequada, uma vez que esse método não estabelece o tipo e a magnitude das diferenças entre um valor padrão e um valor previsto por modelos de estimativa ou outros mecanismos de medida diferentes do padrão (BARROS et al., 2009). O índice de concordância (d) observado na reta tracejada das Figuras 2, 3, 4 e 5, expressa uma medida de exatidão do modelo, o coeficiente de correlação (r), que expressa uma medida da precisão do modelo, e o índice "c", que expressa um desempenho conjunto do modelo, também considerado um indicador de confiança, foram os critérios de indicação dos métodos empíricos a ser utilizado na região.

No Quadro 2 estão apresentados as médias da ETo, o erro padrão de estimativa (EPE), o coeficiente de correlação (r), o índice de concordância (d) e o desempenho (c) e a classificação dos métodos, segundo Camargo e Sentelhas (1997). Os menores EPE foram encontrados pelo método de Hargreaves-Samani $\left(0,917 \mathrm{~mm} \mathrm{dia}^{-1}\right)$ e PriestleyTaylor $\left(0,918 \mathrm{~mm} \mathrm{dia}^{-1}\right)$. Assim, verifica-se que os métodos que utilizam a radiação solar, com exceção do Priestley-Taylor e FAo-24 Radiação obtiveram altos EPE (Quadro 2).

Todos os métodos de estimativa de ETo que não utilizam radiação solar apresentaram "c" abaixo de 0,65 , portanto, seu uso não é recomendado para a região de Aquidauana no solstício de verão. Entre os métodos que utilizam a radiação solar, somente os métodos de Priestley-Taylor e FAO-24 Radiação apresentaram índices satisfatório para seu uso na estação de verão, sendo classificado como "Ótimo" e "Muito bom", respectivamente. O que contribuiu para ambos os métodos apresentarem alta eficiência, foi a utilização da relação entre a declinação da curva de saturação da pressão de vapor de água e a constante psicrométrica em sua equação, que, segundo Allen et al (1998), é o termo energético que contribui com o saldo de energia para a evaporação, que neste caso, é responsável por $78 \%$ da evapotranspiração no solstício de verão de Aquidauana, que também, associados a coeficientes baseados em condições mínimas de advecção, (PEREIRA et al., 1997) obtiveram "d" próximo a linha de exatidão como pode ser observado na Figura 2. 
Quadro 2. Média e Erro Padrão de Estimativa (EPE), coeficiente de correlação (r), índice de concordância (d) e desempenho (c) dos métodos de ETo, em Aquidauana, MS, no solstício de verão

\begin{tabular}{lcccccc}
\hline Métodos* & Média $\left(\mathrm{mm} \mathrm{dia}^{-1}\right)$ & EPE $\left(\mathrm{mm} \mathrm{dia}^{-1}\right)$ & $\mathrm{r}$ & $\mathrm{d}$ & $\mathrm{c}$ & Desempenho \\
\hline PM & 5,168 & - & - & - & - & \\
\hline BL & 5,73 & \multicolumn{2}{c}{ Sem radiação solar } \\
CM & 1,050 & 0,708 & 0,732 & 0,518 & Sofrível \\
HA & 4,84 & 1,206 & 0,383 & 0,331 & 0,127 & Péssimo \\
HS & 4,49 & 1,317 & 0,438 & 0,398 & 0,174 & Péssimo \\
KF & 5,34 & 0,917 & 0,694 & 0,782 & 0,543 & Sofrível \\
\hline & 5,42 & 1,146 & 0,478 & 0,422 & 0,202 & Péssimo \\
\hline JH & & Com radiação solar & & & \\
MK & 7,48 & 2,495 & 0,989 & 0,632 & 0,625 & Bom \\
PT & 7,54 & 2,537 & 0,980 & 0,615 & 0,603 & Mediano \\
RS & 6,02 & 0,918 & 0,986 & 0,899 & 0,887 & Ótimo \\
TR & 6,35 & 1,398 & 0,985 & 0,831 & 0,819 & Muito Bom \\
\hline
\end{tabular}

*PM (Penman-Monteith), BL (Benevides-Lopez), CM (Camargo), HA (Hamon), HS (Hargreaves-Samani), KF (Klarrufa), JH (Jensen-Haise), MK (Makink), PT (Priestley-Taylor), RS (FAO-24 Radiação) e TR (Turc).

Conceição e Mandelli (2005) avaliaram a adequabilidade de métodos que empregam a temperatura do ar ou radiação solar como variáveis de entrada comparando-os aos valores diários estimados pelo método padrão Penman-Monteith para a região de Bento Gonçalves-RS e observaram que os melhores resultados foram obtidos com os métodos que empregam a radiação global incidente como variável na equação. $\mathrm{O}$ mesmo foram observado por Pereira et al. (2009), na região da Serra da Mantiqueira, no qual destacaram que os métodos de estimativa da evapotranspiração de referência que empregam radiação solar apresentam melhor desempenho que os métodos que utilizam apenas a temperatura do ar.

No equinócio de Outono, os métodos Camargo, Hamon e Hargreaves-Samani apresentaram coeficiente "b" de 1,046; 1,096 e 1,015, respectivamente, indicando previamente ótima performance na resposta em estimativas de ETo comparado com o método padrão PenmanMonteith (Figura 3). Contudo, o método de
Hargreaves-Samani comparado aos modelos que não utilizam radiação solar incidente foi o que apresentou maior precisão do modelo de regressão com $\mathrm{r}^{2}$ de 0,733 . Os métodos de Benevides-Lopez, Camargo, Hamon e Klarrufa obtiveram $r^{2}$ de 0,577; 0,$574 ; 0,569$ e 0,456 , respectivamente, sendo que o método de Benevides-Lopez e Klarrufa apresentaram tendência em superestimar a ETo no equinócio de outono em Aquidauana, MS.

Analisando o coeficiente " $b$ ", dos métodos que utilizam a radiação solar, verificam-se que todos apresentaram tendência em superestimar a ETo estimado pelo método padrão Pemnam-Monteith. Porém, todos ajustaram-se expressivamente ao modelo de regressão com pouca dispersão, apresentando altos valores de $\mathrm{r}^{2}$ (Figura 3). Logo, o método de Priestley-Taylor apresentou o melhor ajuste entres os dados em pares, apresentando ótimos ajustes, que pode ser verificado na Figura 3 , que mostra coeficiente "b" e $\mathrm{r}^{2}$ igual a 0,788 e 0,931 , respectivamente. 

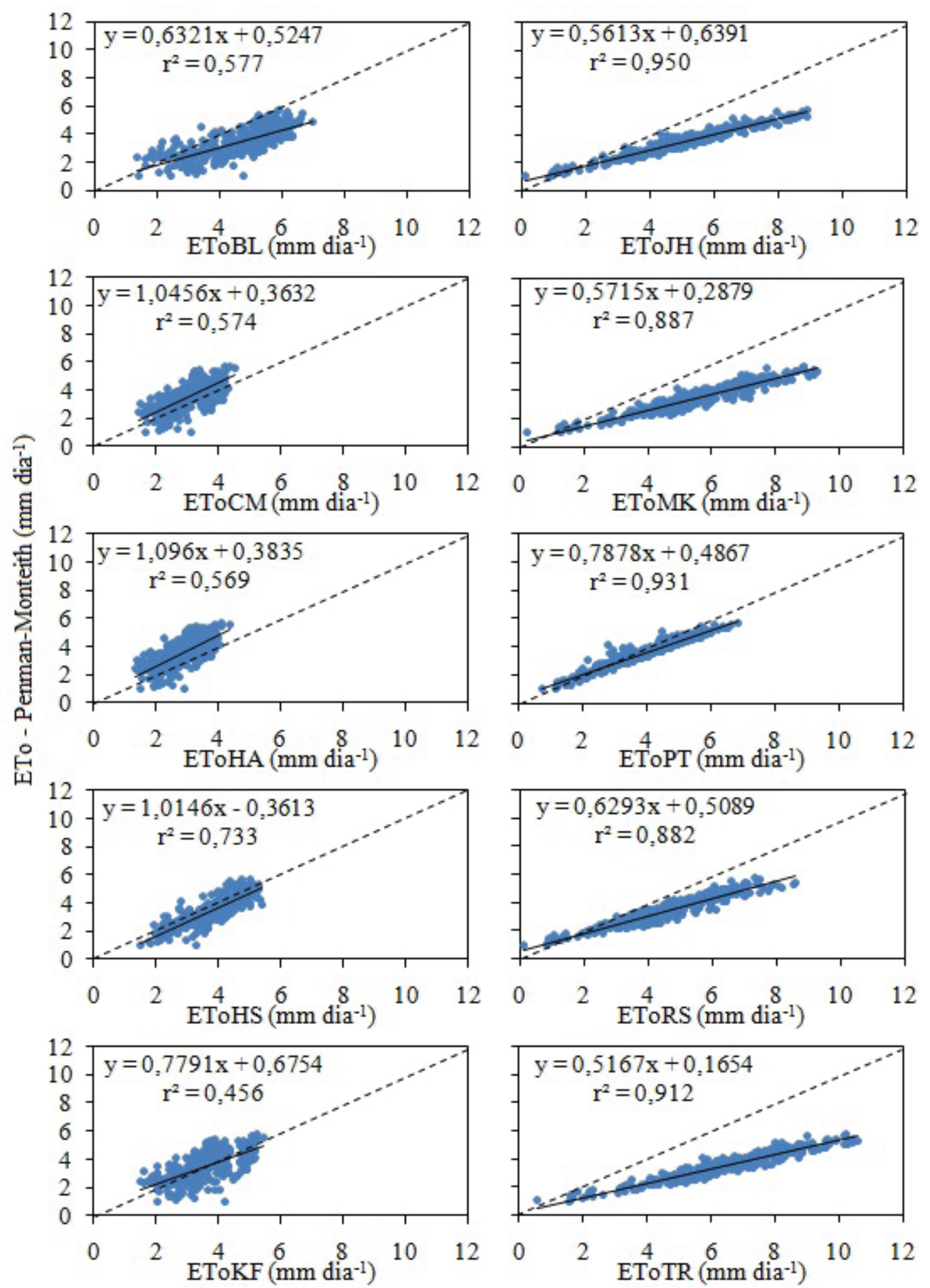

Figura 3. Coeficiente de determinação entre os valores diários de evapotranspiração de referência (ETo) estimados pelo método padrão Penman-Monteith e os métodos empíricos, no equinócio de outono de Aquidauana, MS. BL (Benevides-Lopez), CM (Camargo), HA (Hamon), HS (HargreavesSamani), KF (Klarrufa), JH (Jensen-Haise), MK (Makink), PT (Priestley-Taylor), RS (FAO-24 Radiação) e TR (Turc). A linha tracejada indica a reta 1:1. 
No Quadro 3, observa-se que entre os métodos que não utilizam radiação solar, o método de Hargreaves-Samani foi o único que alcançou índice "c" (0,760) suficiente para ser aplicado às condições do equinócio de outono de Aquidauana, sendo o mesmo classificado como "Muito Bom". Nesta estação, o método de Hargreaves-Samani apresentou média de ETo de $3,74 \mathrm{~mm} \mathrm{dia}^{-1}$, ou seja, uma variação com cerca de 0,31 $\mathrm{mm} \mathrm{dia}^{-1}$ e, EPE na ordem de 0,602 mm $\mathrm{dia}^{-1}$, sendo que sua correlação $\mathrm{r}$ e índice " $\mathrm{d}$ " obtiveram valores muito próximos, contribuindo para seu bom desempenho neste período.

Os melhores métodos baseados na radiação solar no equinócio de outono foram o PriestleyTaylor seguido de FAO-24 Radiação e JensenHaise, no qual foram classificados como "Ótimo" e "Bom", respectivamente. O método de Priestley-Taylor apresentaram excelentes resultados estatísticos com o menor EPE $(0,481$ mm dia-1), e maior "r", "d" e "c", com valores de 0,965, 0,953 e 0,920, respectivamente.

Apesar do método Makink e Turc utilizarem a radiação solar em sua equação de estimativa da ETo, os mesmos apresentaram baixa performance, com índices "d" muito baixos, contribuindo para seu desempenho não satisfatório, sendo que esse fato pode ser observado na Figura 3, onde a reta do modelo de regressão esta bastante abaixo da reta de exatidão.

No solstício de inverno os métodos que não utilizam radiação solar incidente; BenevidesLopez, Camargo, Hamon, Hargreaves-Samani e Klarrufa obtiveram $\mathrm{r}^{2}$ de 0,$649 ; 0,610 ; 0,582$; 0,629 e 0,523, respectivamente (Figura 4). Estes métodos apresentaram maior precisão dos modelos, contudo, o método que apresentou maior tendência em superestimar a ETo foi o Benevides-Lopez.

Verifica-se que os métodos Hargreaves-

Quadro 3. Média e Erro Padrão de Estimativa (EPE), coeficiente de correlação (r), índice de concordância(d) e desempenho (c) dos métodos de ETo, em Aquidauana, MS, no equinócio de outono

\begin{tabular}{lcccccc}
\hline Métodos & Média $\left(\mathrm{mm} \mathrm{dia}^{-1}\right)$ & $\mathrm{EPE}\left(\mathrm{mm} \mathrm{dia}{ }^{-1}\right)$ & $\mathrm{r}$ & $\mathrm{d}$ & $\mathrm{c}$ & Desempenho \\
\hline PM & 3,431 & - & - & - & - \\
\hline BL & \multicolumn{1}{c}{ Sem radiação solar } & & \\
CM & 4,60 & 1,409 & 0,759 & 0,648 & 0,492 & Mau \\
HA & 2,93 & 0,821 & 0,758 & 0,765 & 0,580 & Sofrível \\
HS & 2,78 & 0,927 & 0,755 & 0,708 & 0,534 & Sofrível \\
KF & 3,74 & 0,602 & 0,856 & 0,889 & 0,760 & Muito bom \\
\hline & 3,54 & 0,769 & 0,676 & 0,798 & 0,539 & Sofrível \\
\hline JH & & Com radiação solar & & & Bom \\
MK & 4,97 & 1,737 & 0,974 & 0,692 & 0,674 & Sofrível \\
PT & 5,50 & 2,215 & 0,942 & 0,559 & 0,526 & Ótimo \\
RS & 3,74 & 0,481 & 0,965 & 0,953 & 0,920 & Bom \\
TR & 4,64 & 1,378 & 0,939 & 0,747 & 0,702 & Mau \\
\hline
\end{tabular}

*PM (Penman-Monteith), BL (Benevides-Lopez), CM (Camargo), HA (Hamon), HS (Hargreaves-Samani), KF (Klarrufa), JH (Jensen-Haise), MK (Makink), PT (Priestley-Taylor), RS (FAO-24 Radiação) e TR (Turc). 

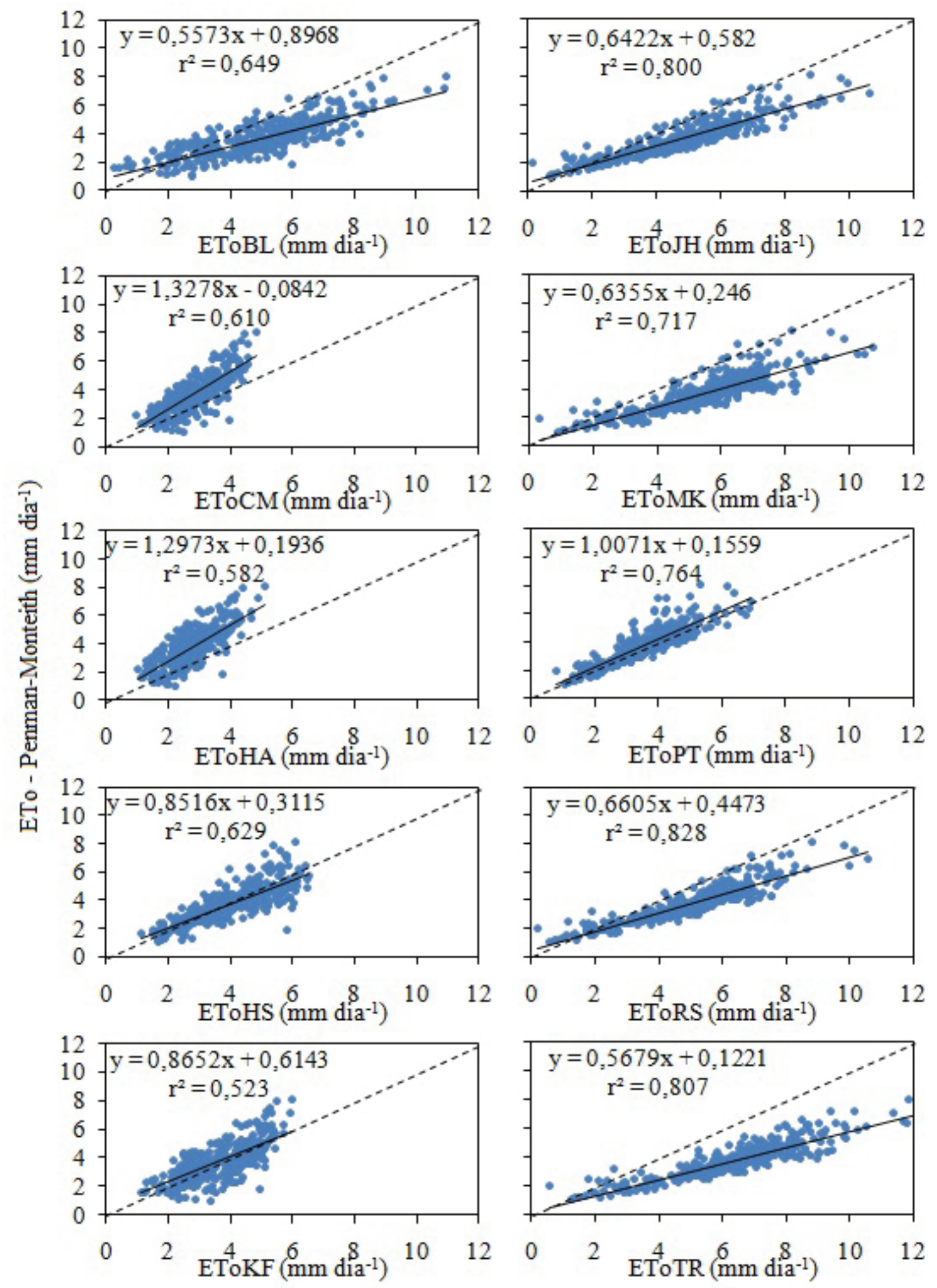

Figura 4. Coeficiente de determinação entre os valores diários de evapotranspiração de referência (ETo) estimados pelo método padrão Penman-Monteith e os métodos empíricos, no solstício de inverno de Aquidauana, MS. BL (Benevides-Lopez), CM (Camargo), HA (Hamon), HS (HargreavesSamani), KF (Klarrufa), JH (Jensen-Haise), MK (Makink), PT (Priestley-Taylor), RS (FAO-24 Radiação) e TR (Turc). A linha tracejada indica a reta 1:1. 
Samani e Klarrufa apresentaram "b" próximo de 1, ou seja, a distribuição da ETo em relação ao método padrão Penman-Monteith está superestimada em, aproximadamente, 15\%. Apesar desta superestimativa, destaca-se o método de Hargreaves-Samani por utilizar somente dados de temperatura do ar como parâmetro de entrada do método. Talvez, essa melhora geral da precisão dos métodos que não utilizam radiação solar, esteja ligada a menor incidência de radiação solar do Hemisfério Sul no solstício de inverno devido, principalmente, ao menor tempo de luz e maior regularidade (distribuição homogênea) da temperatura do ar e umidade relativa do ar, sendo esse período, para a região de Aquidauana, MS, correlacionado com períodos de baixa nebulosidade e chuva.

Entre os métodos baseados em radiação solar para o solstício de inverno, observa-se uma diminuição do $\mathrm{r}^{2}$ quando comparado com o solstício de verão, no entanto indicando boa precisão da equação de regressão, $\operatorname{com~r}^{2}$ de 0,800 ; 0,717; 0,764; 0,828 e 0,807 para os métodos de Jensen-Haise, Makink, Priestley-Taylor, FAO-24 Radiação e Turc, respectivamente, com destaque ao método de Priestley-Taylor que apresenta "b" próximo de 1 , indicando preliminarmente, ótima estimativa de ETo quando comparado com o método padrão Penman-Monteith (Figura 4).

O saldo de radiação utilizado pelo método de Priestley-Taylor promoveu essa menor variação em relação ao "b", sendo que o método padrão Penman-Monteith também utiliza o saldo de radiação e essa semelhança contribuiu para essa maior aproximação. Os demais métodos, por meio do modelo de regressão previsto pelo $\mathrm{r}^{2}$, condicionaram tendência em superestimar a ETo de referência como pode ser observado na Figura 4, onde todos esses métodos apresentaram semelhança principalmente pelo fato de todos usarem a radiação solar.

Em Campina Grande, PB, Henrique e Dantas (2007), estudando o método de Hargreaves-
Samani e comparando ao padrão PenmanMonteith no solstício de inverno, encontraram coeficientes "b" de 1,054 e r ${ }^{2}$ de 0,878 , destacando como o melhor entre os métodos que não utilizam radiação solar. Em estudo com comparação de métodos de estimativa de ETo no município de Juazeiro, BA, Oliveira et al. (2010) observaram que o método Hargreaves-Samani apresentou o melhor desempenho, com "c" $=0,67$ e, Guedes Filho et al. (2011) estudando a correlação de métodos de ETo no município de Areia, PB, verificaram que o método Hargreaves-Samani mostrou coeficiente de determinação de $95 \%$ quando correlacionado com o método padrão.

Entre os métodos que não utilizam radiação solar, destaca-se o Hargreaves-Samani, por atingir índice de desempenho satisfatório $(0,69)$ para o seu uso, sendo classificado como "Bom" (Quadro 4). Vale ressaltar que o mesmo utiliza somente dados de temperatura do ar, sendo facilmente utilizado por qualquer produtor irrigante que não dispõe de dados de radiação solar. O método de Hargreaves-Samani apresentou média de ETo de $4,01 \mathrm{~mm} \mathrm{dia}^{-1}$, muito próximo ao método padrão Penman-Monteith, com bom " $r$ " $(0,793)$, sendo que o principal fator para elevação do índice "c" foi o índice "d" $(0,870)$, acima de vários métodos que utilizam radiação solar e pode ser observado que a reta da equação esta bastante próximo a reta de exatidão (linha tracejada) (Figura 4).

Os métodos que utilizam radiação solar Jensen-Haise, Priestley-Taylor e FAO-24 Radiação obtiveram classificação de "Bom, Muito bom e Bom", respectivamente. Nota-se que entre esses modelos o método de PriestleyTaylor obteve ETo média muito próxima de método padrão Penman-Monteith com diferença na ordem de $-0,181 \mathrm{~mm} \mathrm{dia}^{-1}$ com menor EPE $\left(0,625 \mathrm{~mm} \mathrm{dia}^{-1}\right)$ e índice de "d" 0,922 (Quadro 4). Segundo Tazzo et al. (2012), a estimativa da ETo por meio de modelos que utilizam variáveis de insolação, temperatura do ar, são menos precisos do que as que possuem aporte de energia, no entanto as variáveis de energia 
Quadro 4. Média e Erro Padrão de Estimativa (EPE), coeficiente de correlação (r), índice de concordância (d) e desempenho (c) dos métodos de ETo, em Aquidauana, MS, no solstício de inverno

\begin{tabular}{lcccccc}
\hline Métodos & Média $\left(\mathrm{mm} \mathrm{dia}^{-1}\right)$ & EPE $\left(\mathrm{mm} \mathrm{dia}^{-1}\right)$ & $\mathrm{r}$ & $\mathrm{d}$ & $\mathrm{c}$ & Desempenho \\
\hline PM & 3,727 & - & - & - & - & - \\
\hline BL & 5,08 & 1,729 & 0,805 & 0,703 & 0,566 & Sofrível \\
CM & 2,87 & 1,177 & 0,781 & 0,669 & 0,522 & Sofrível \\
HA & 2,72 & 1,301 & 0,763 & 0,617 & 0,471 & Mau \\
HS & 4,01 & 0,821 & 0,793 & 0,870 & 0,690 & Bom \\
KF & 3,60 & 0,872 & 0,723 & 0,829 & 0,599 & Sofrível \\
\hline & & Com radiação solar & & & \\
\hline JH & 4,90 & 1,433 & 0,894 & 0,787 & 0,704 & Bom \\
MK & 5,48 & 1,965 & 0,847 & 0,640 & 0,542 & Sofrível \\
PT & 3,55 & 0,625 & 0,874 & 0,922 & 0,806 & Muito Bom \\
RS & 4,96 & 1,460 & 0,910 & 0,781 & 0,711 & Bom \\
TR & 6,35 & 2,809 & 0,898 & 0,523 & 0,469 & Mau \\
\hline
\end{tabular}

*PM (Penman-Monteith), BL (Benevides-Lopez), CM (Camargo), HA (Hamon), HS (Hargreaves-Samani), KF (Klarrufa), JH (Jensen-Haise), MK (Makink), PT (Priestley-Taylor), RS (FAO-24 Radiação) e TR (Turc).

possuem maior dificuldade para sua obtenção, por serem mais caros e não estarem presentes em todos os postos meteorológicos.

Os métodos baseados basicamente em temperatura do ar no equinócio de primavera mostraram coeficiente "b" próximo de 1 , com exceção do método Camargo, que de acordo com o modelo de regressão, apresenta tendência em subestimar a ETo em $38 \%$ quando comparado com o método padrão. O método de BenevidesLopez obteve "b" de 0,807 , ou seja, subestimando a ETo em 19,3\%, com precisão da estimativa de $52,7 \%$, mostrado pelo $\mathrm{r}^{2}$ (Figura 5).

$\mathrm{O}$ método Hamon foi o que encontrou o melhor coeficiente angular $(1,024)$, porém, péssima precisão do modelo de regressão $\left(\mathrm{r}^{2}=\right.$ $0,313)$. O maior $\mathrm{r}^{2}$ foi encontrado com método de Hargreaves-Samani, explicado em 61\% através do modelo de regressão, isso referente a distribuição dos dados correlacionados com os valores de ETo do método padrão.
Torna-se importante salientar que o método de Camargo e Hargreaves-Samani, apresenta em comum, o uso da radiação no topo da atmosfera, porém, os coeficientes técnicos do método de Hargreaves-Samani e o uso de um conjunto de relação entre a temperatura do ar, como temperatura média mínima e máxima do ar, faz com que esse método seja mais eficiente que o método de Camargo, que utiliza apenas a temperatura média do ar e coeficiente de ajuste. Como apresentado anteriormente, os métodos que utilizam radiação solar novamente obtiveram os maiores valores de $\mathrm{r}^{2}$ (Figura 5 ).

Ainda na Figura 5, verifica-se que o método de Priestley-Taylor superestima a ETo em, aproximadamente, $20 \%$, sendo que o modelo de regressão apresenta precisão próximo a $90 \%$. Entre as estações do ano, verificou-se que o menor $\mathrm{r}^{2}$ foi com utilização do método de Priestley-Taylor, encontrado na estação de inverno. 

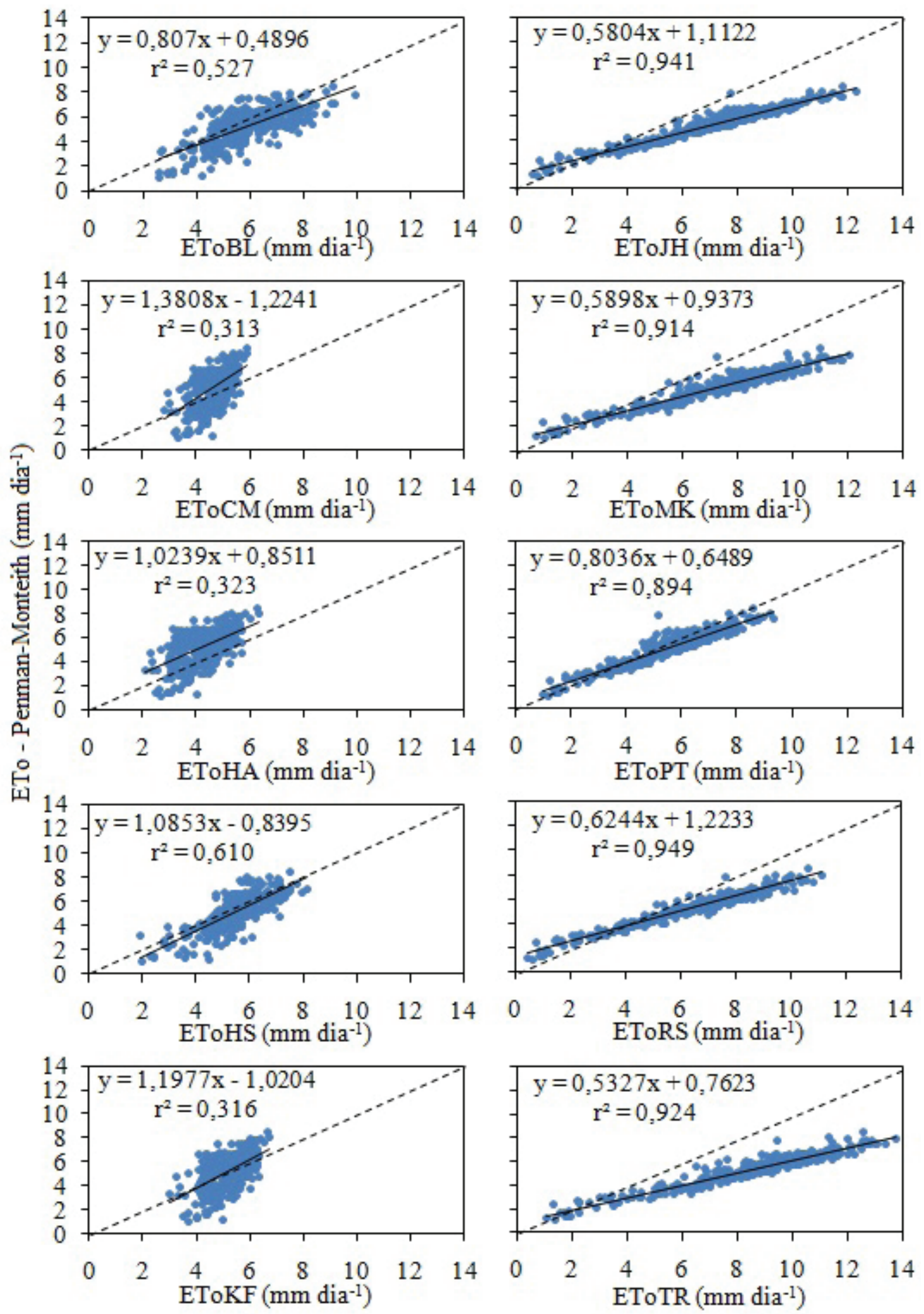

Figura 5. Coeficiente de determinação entre os valores diários de evapotranspiração de referência (ETo) estimados pelos métodos de Penman-Monteith e os métodos empíricos, no equinócio de primavera de Aquidauana, MS. BL (Benevides-Lopez), CM (Camargo), HA (Hamon), HS (Hargreaves-Samani), KF (Klarrufa), JH (Jensen-Haise), MK (Makink), PT (Priestley-Taylor), RS (FAO-24 Radiação) e TR (Turc). 
Quadro 5. Média e Erro Padrão de Estimativa (EPE), coeficiente de correlação (r),índice de concordância(d) e desempenho (c) dos métodos de ETo, em Aquidauana, MS, no equinócio de primavera

\begin{tabular}{lcccccc}
\hline Métodos & Média $\left(\mathrm{mm} \mathrm{dia}^{-1}\right)$ & EPE $\left(\mathrm{mm} \mathrm{dia}^{-1}\right)$ & $\mathrm{r}$ & $\mathrm{d}$ & $\mathrm{c}$ & Desempenho \\
\hline PM & 5,261 & - & - & - & - & \\
\hline BL & 5,91 & 1,190 & 0,726 & 0,784 & 0,569 & Sofrível \\
CM & 4,70 & 1,311 & 0,559 & 0,509 & 0,284 & Péssimo \\
HA & 1,498 & 0,568 & 0,525 & 0,298 & Péssimo \\
HS & 4,31 & 0,951 & 0,781 & 0,830 & 0,648 & Mediano \\
KF & 5,62 & 1,167 & 0,562 & 0,604 & 0,340 & Péssimo \\
\hline & 5,25 & Com radiação solar & & & \\
\hline JH & 7,15 & 2,158 & 0,970 & 0,733 & 0,711 & Bom \\
MK & 7,33 & 2,310 & 0,956 & 0,696 & 0,665 & Bom \\
PT & 5,74 & 0,737 & 0,946 & 0,942 & 0,890 & Ótimo \\
RS & 6,47 & 1,494 & 0,974 & 0,843 & 0,821 & Muito Bom \\
TR & 8,45 & 3,424 & 0,961 & 0,538 & 0,517 & Sofrível \\
\hline
\end{tabular}

*PM (Penman-Monteith), BL (Benevides-Lopez), CM (Camargo), HA (Hamon), HS (Hargreaves-Samani), KF (Klarrufa), JH (Jensen-Haise), MK (Makink), PT (Priestley-Taylor), RS (FAO-24 Radiação) e TR (Turc).

Os métodos Benevides-Lopez, Camargo, Hamon, Hargreaves-Samani, Klarrufa, e Turc não atingiram índice de desempenho suficiente para ser utilizado em Aquidauana, no equinócio de primavera, sendo que os mesmos foram classificados como "Mediano, Péssimo, Péssimo, Sofrível, Péssimo, e Sofrível", respectivamente, em relação aos métodos (Quadro 5).

Os métodos de Jensen-Haise, Makkink, Priestley-Taylor e FAO-24 Radiação, podem ser utilizados na região de Aquidauana para estimar a ETo, sendo classificado como "Bom, Bom, Ótimo e Muito bom", respectivamente.

A maior diferença de média da ETo foi de $3,19 \mathrm{~mm} \mathrm{dia}^{-1}$, obtida pelo método de Turc, que apresentou EPE de 3,424 $\mathrm{mm} \mathrm{dia}^{-1}$. Verifica-se que em todas as estações do ano este método foi o que apresentou a maior média da ETo. O método Priestley-Taylor no equinócio de primavera apresentou melhores índices estatísticos, como se observa no Quadro 5.

\section{CONCLUSÕES}

- Diante dos resultados obtidos entende-se que os métodos Priestley-Taylor e FAO-24 Radiação atendem satisfatoriamente à estimativa da evapotranspiração de referência na região de Aquidauana, MS, para todas as épocas do ano;

- Dentre os métodos que utilizam a temperatura como base, apenas o Hargreaves-Samani é recomendado para o solstício de inverno e equinócio de outono;

- O método Jensen-Haise, não é recomendado para o solstício de verão e o método Makkink pode ser utilizado apenas no equinócio de primavera para esta série de dados.

\section{REFERÊNCIAS BIBLIOGRÁFICAS}

ALLEN, R.G.; PEREIRA, L.S.; RAES, D.; SMITH, M. Crop evapotranspiration - Guidelines for computing crop water requirements. Roma: 
FAO, 1998. 300p. (FAO Irrigation and Drainage Paper, 56).

ARAÚJO, W.F.; COSTA, S.A.A.; SANTOS, A.E. Comparação entre métodos de estimativa da evapotranspiração de referência (ETo) para Boa Vista, RR. Caatinga, Mossoró, v.20, n.4, p.84-88, 2007.

BARROS, V.R.; SOUZA, A.P.; FONSECA, D.C.; SILVA, L.B.D. Avaliação da evapotranspiração de referência na Região de Seropédica, Rio de Janeiro, utilizando lisímetro de pesagem e modelos matemáticos. Revista Brasileira de Ciências Agrárias, Recife, v.4, n.2, p.198-203, 2009.

BENEVIDES, J.G.; LOPEZ DIAZ, J. Formula para el calculo de la evapotranspiracion potencial adaptada al tropico $\left(15^{\circ} \mathrm{N}-15^{\circ} \mathrm{S}\right)$. Agronomia Tropical, Maracay, v.20, n.5, p.335-345, 1970.

CAMARGO, A.P.; SENTELHAS, P.C. Avaliação do desempenho de diferentes métodos de estimativa da evapotranspiração potencial no Estado de São Paulo, Brasil. Revista Brasileira de Agrometeorologia, Santa Maria, v.5, n.1, p.89-97, 1997.

CONCEIÇÃO, M.A.F.; MANDELLI, F. Comparação entre métodos de estimativa da evapotranspiração de referência em Bento Gonçalves, RS. Revista Brasileira de Agrometeorologia, Santa Maria, v.13, n.2, p.303307, 2005.

GUEDES FILHO, D.H.; SANTOS JÚNIOR, J.A.; COSTA FILHO, J.F.; FRANCISCO, P.R.M.; CAMPOS, V.B. Estimativa da evapotranspiração de referência para a cidade de Areia, Paraíba. Revista Brasileira de Agricultura Irrigada, Fortaleza, v.5, n.1, p.37-47, 2011.

HENRIQUE, F.A.N.; DANTAS, R.T. Estimativa da evapotranspiração de referência em Campina Grande, Paraíba. Revista Brasileira de Engenharia Agrícola e Ambiental, Campina Grande, v.11, n.6, p.594-599, 2007.

JENSEN, M.E.; HAISE, H.R. Estimating evapotranspiration from solar radiation. Journal of Irrigation Drainage Division, ASCE, New York, v.89, n.1, p.15-41, 1963.

KLARRUFA, N.S. Simplified equation for evapotranspiration in arid regions. Beiträgezur Hydrologie, Kirchzarten, Sonderheft, v.5, n.1, p.39-47, 1985.

OLIVEIRA， G.M.; LEITÃO, M.M.V.B.R.; BISPO, R.C.; SANTOS, I.M.S.; ALMEIDA, A.C. Comparação entre métodos de estimativa da evapotranspiração de referência na região Norte da Bahia. Revista Brasileira de Agricultura Irrigada, Fortaleza, v.4, n.2, p.104-109, 2010.

PEREIRA, A.R.; VILLA NOVA, N.A.; SEDIYAMA, G.C. Evapo(transpi)ração. Piracicaba: Fealq, 1997, 183p.

PEREIRA, D.R.; YANAGE, S.N.M.; MELLO, C.R.; SILVA, A.M.; SILVA, L.A. Desempenho de métodos de estimativa da evapotranspiração de referência para a região da Serra da Mantiqueira, MG. Ciência Rural, Santa Maria, v.39, n.9, p.2488-2493, 2009.

SMITH, M. Report on the expert consultation on procedures for revision of FAO guidelines for prediction of crop water requirements: RomFAO, 1991, 54p.

SOUSA, I.F.; SILVA, V.P.R.; SABINO, F.G.; NETTO, A.O.; SILVA, B.K.N.; AZEVEDO, P.V. Evapotranspiração de referência nos perímetros irrigados do estado de Sergipe. Revista Brasileira de Engenharia Agrícola e Ambiental, Campina Grande, v.14, n.6, p.633-644. 2010.

SILVA, A.P.N.; SOUZA, L.R. Estimativa de evapotranspiração de referência no Semiárido Pernambucano. Engenharia Ambiental, Espírito Santo do Pinhal, v.8, n.4, p.3-22, 2011.

TAZZO, I.F.; HELDWEIN, A.B.; MALDANER, I.C.; PIVETTA, C.R.; STRECK, L.; RIGHI, E.Z. Evapotranspiração do pimentão em estufa plástica estimada com dados meteorológicos externos, na primavera. Revista Brasileira de Engenharia 
Agrícola e Ambiental, Campina Grande, v.16, n.3, p.275-280, 2012.

TURC, L. Estimation of irrigation water requirements, potential evapotranspiration: a simple climatic formula evolved up to date. Annales Agronomique, Paris, v.12, p.13-49, 1961.

WILLMOT, C.J.; ACKLESON, S.G.; DAVIS, J.J.; FEDDEMA, K.; KLINK, D.R. Statistics for the evaluation and comparison of models. Journal of Geophysical Research, Ottawa, v.90, n.5, p.89959005, 1985.

ZAVATTINI, J.A. As chuvas e as massas de ar no estado de Mato Grosso do Sul: estudo geográfico com vista à regionalização climática São Paulo: Editora UNESP; São Paulo: Cultura Acadêmica, 2009. 212. Disponível em: < http://books.scielo. org>. Acesso em: 05 out. 2013. 